\title{
Smart Car Parking System using Arduino UNO
}

\author{
Suvarna Nandyal, $\mathrm{PhD}$ \\ Prof \& H.O.D Dept \\ Of Computer Science \& \\ Engineering \\ PDA College of Engineering \\ Gulbarga, India
}

\author{
Sabiya Sultana \\ Student \\ Computer Science \& \\ Engineering Department \\ PDA College of Engineering \\ Gulbarga, India
}

\author{
Sadaf Anjum \\ Student \\ Computer Science \& Engineering \\ Department \\ PDA College of Engineering \\ Gulbarga, India
}

\begin{abstract}
In the early times the concept of smart cities have gained great popularity. The proposed Smart Parking system consists of an on-site deployment of an IOT module that is used to monitor and signalize the state of availability of single parking space. This paper introduce an IOT based coordinated framework for efficient and easy way of parking the vehicles by checking the availability of slots. The proposed Smart Parking framework comprises of an IOT module that is utilized to screen and signalize the condition of accessibility of single parking spot. The paper additionally depicts an abnormal state perspective of the framework engineering. Towards the end, the paper examines the working of the framework in type of an utilization case that demonstrates the rightness of the proposed show.
\end{abstract}

The Ultrasonic Range Detection Sensor is utilized with Arduino to indicate the empty slot .By measuring the distance using ultrasonic sensor drivers are able to find the empty slot in parking to park the car and help the driver to find the slot easily and reduce the searching time.As the parking place is found to be empty it is detected using ultrasonic sensors which report it further. We achieved this by programming the sensors and Arduino.

\section{Keywords}

Smart Car Parking, IOT, Ardunio Uno, Ultrasonic Sensor.

\section{INTRODUCTION}

At the point when IoT is increased with sensors and actuators, the innovation turns into an occurrence of the more broad class of digital physical frameworks, which likewise incorporates advances. For Example, keen networks, virtual power plants, brilliant homes, astute transportation and shrewd urban communities. Among the difficulties that confront in everyday life one of most unavoidable test is parking the car wherever people go. As our need expands our setting out increments however because of extreme increment in utilization of vehicles and increment in populace this project confront the intense assignment of parking car especially amid busiest hours of the day. Amid pinnacle hours the majority of the saved parking zone gets full and this leaves the client to scan for their parking among other parking area which makes more movement and abandons them with no sign on accessibility of parking spot. To defeat this issue there is certainly a requirement for composed parking in business condition. To outline such parking there need to assess reservation of parking space with ideal parking spot which relies upon cost and time. However this project compose the time driven grouping strategy which takes care of the issue of parking utilizing opening assignment technique.
The fundamental inspiration of this venture is to diminish the movement clog that happens in and around the urban zones which is brought on by vehicles looking for parking. In the daily papers, many articles with respect to the stopping issue all over India like Delhi, Mumbai, Chennai, Bangalore and numerous metropolitan urban areas. Developing populace has made numerous issues; stopping issue is one of the enormous issues in our everyday life. In a current study, analysts have found that for one year, car cruising for stopping made what might as well be called 38 times trips far and wide, consuming 177914.8 liters of fuel and delivering 730 tons of $\mathrm{CO} 2$. To diminish every one of these elements we go for the savvy stopping framework.

a) To build up a canny, easy to understand robotized car stopping framework which diminishes the labour and movement blockage.

b) To offer sheltered and secure stopping openings inside constrained territory.

\section{Parking garage Problems}

Trouble in Finding Vacant Spaces, Quickly finding an empty space in a multilevel parking garage is troublesome if not unthinkable, particularly on ends of the week or open occasions. Discovering spaces amid ends of the week or open occasions can take over 10 minutes for around $66 \%$ of guests. Stadiums or shopping center are swarmed at pinnacle periods, and trouble in finding empty openings at these spots is a noteworthy issue for clients. Inadequate car parking space 1 prompt activity blockage and driver disappointment.

\section{RELATED WORK}

2.1 Mr. Basavaraju S R-2015 has proposed An Carmatic Smart Parking System using Internet of Things (IOT)-------Internet of Things (IOT) plays a vital role in connecting the surrounding environmental things to the network and made easy to access those un-internet things from any remote location. It's inevitable for the people to update with the growing technology. And generally people are facing problems on parking vehicles in parking slots in a city. In this study we design a Smart Parking System (SPS) which enables the user to find the nearest parking area and gives availability of parking slots in that respective parking area. And it mainly focus on reducing the time in finding theparking lots and also it avoids the unnecessary travelling through filled parking lots in a parking area. Thus it reduces the fuel consumption which in turn reduces carbon footprints in an atmosphere.

Our system is a Raspberry pi based parking sensor which contains pi-camera to detect the empty parking spaces and sends this data to server, this stored data is accessed by users. 
This enhances the user to check the status/availability of parking spaces before setting their journey. Here the challenge is to use the existing resources in optimum level to reduce the searching time, traffic congestion in the city. Some embedded systems such as auridino, raspberry pi, Tsgate, Tsmote etc. are used to develop internet of things applications.

A few existing parking system which uses sensors to collect the information but using sensors like video sensors in a parking system are expensive so our aim is to develop a system with less cost with more performance.

\subsection{Deepthi. S, Anil A R has proposed A} Survey on Smart Parking System Based on Internet of Things------ In today life people don't depend on public vehicles. They use their own vehicles to travel. So traffic increases. When people travel through a city the most difficult problem is to park the vehicle. It causes not only a waste of time and fuel for drivers looking for parking but it also leads to additional waste of time and fuel for other drivers as a result of traffic congestion. At first we use PGI (Parking Guidance Information) for better parking management. Parking information may be displayed on VMS (Variable Message Sign) at major roads or streets or it may be disseminated through the internet. In PGI systems e-parking is an innovative platform which allows drivers to obtain parking information before or during a trip and reserve a parking spot.

2.2.1 To overcome the limitations of PGI system Yanfeng Geng proposes [3] a new concept "Optimal parking based on resource allocation and reservation". It uses the concept of mixed integer linear problem. When people go through a downtown area, there much rush and traffic. In this case to find the available location for parking is very difficult. So implementing optimal parking people can easily park vehicle in the reserved locations. When a person with disability trying to park a vehicle in a city having heavy traffic, So he faces several problems in such a situation to search and park the vehicle difficult. The disabled person can park the vehicle in a specially designed location. The intelligent parking finds the best available parking, minimizing the cost. The parking and un parking can be done with the help of an android application. User has to install the android application on his/her smart phone devices, which would have the facility to pre-book the parking slot.

2.3 D. J. Bonde Jan-2012 has proposed "Carmated car parking system commanded by android application"-----The aim of this project is to carmate the car and car parking as well. A miniature model of an carmated car parking system that can regulate and manage number of cars that can be parked in given space at any given time based on the availability of parking slot. Carmated parking is a method of parking and existing cars using sensing device.

2.4 Abhirup Khanna-2016 has worked on IOT based smart parking system-----Recent times the concept of smart cities have gained grate popularity. Thanks to the evolution of Internet of things the idea of smart city now seems to be achievable. Consistent efforts are being made in the field of IoT in order to maximize the productivity and reliability of urban infrastructure. Problems such as, traffic congestion, limited car parking facilities and road safety are being addressed by IoT. In this paper, we present an IoT based cloud integrated smart parking system. The proposed Smart Parking system consists of an on-site deployment of an IoT module that is used to monitor and signalize the state of availability of each single parking space.

The concept of Smart Cities have always been a dream for humanity. Since the past couple of years large advancements have been made in making smart cities a reality. The growth of Internet of Things and Cloud technologies have give rise to new possibilities in terms of smart cities. Smart parking facilities and traffic management systems have always been at the core of constructing smart cities. In this paper, we address the issue of parking and present an IoT based Cloud integrated smart parking system. The system that we propose provides real time information regarding availability of parking slots in a parking area. Users from remote locations could book a parking slot for them by the use of our mobile application. The efforts made in this paper are indented to improve the parking facilities of a city and thereby aiming to enhance the quality of life of its people.

2.5 R. Yusnita, FarizaNorbaya, and Norazwinawati Basharuddin-2012 has proposed "Intelligent Parking Space Detection System Based on Image Processing"-------This paper aims to present an intelligent system for parking space detection based on image processing technique that capture and process the brown rounded image drawn at parking lot and produce the information of the empty car parking spaces. It will be display at the display unit that consists of seven segments in real time. The seven segments display shows the number of current available parking lots in the parking area. This proposed system, has been developed in software and hardware platform.

2.6 M. M. Rashid, A. Musa, M. AtaurRahman, and N. Farahana, A. Farhana2012 has proposed "Carmatic Parking Management System and Parking Fee Collection Based on Number Plate Recognition"-----This paper discussed on carmatic parking system and electronic parking fee collection based on vehicle number plate recognition. The aim of this research is to develop and implement an carmatic parking system that will increase convenience and security of the public parking lot as well as collecting parking fee without hassles of using magnetic card. The car parking system will able to have less interaction of humans and use no magnetic card and its devices. In additions to that, it has parking guidance system that can show and guide user towards a parking space. The system used image processing of recognizing number plates for operation of parking and billing system. Overall, the systems run with pre-programmed controller to make minimum human involvement in parking system and ensure access control in restricted places.

2.7 D.B.L. Bong, K.C. Ting and K.C. Lai2007 has worked on Integrated approach in the design of car park occupancy information system (coins)----- In large parking areas such as those at mega shopping malls or stadiums, drivers always have difficulty to find vacant car park lots especially during peak periods or when the parking lots are almost full. A solution to reduce the drivers' searching time for vacant car-park lots will greatly save time, reduce cost and improve the traffic flow in the car park areas. In this paper, a research project which was developed to acquire car-park occupancy information using integrated approach of image processing algorithms is 
presented. Security surveillance cameras which are readily available in most car parks can be used to acquire the images of the car park. This project is called as Car-Park Occupancy Information System (COINS), and it was tested using simulation model and also in real-case scenarios. Many different parking guidance systems have been developed and designed to shorten the searching time for vacant parking lots. This is especially crucial for drivers who need to search for available car parks during peak hours or when the car parks are almost full. Researches on car park occupancy information generally fall into four categories - counter-based, wiredbased, wireless-based and image-based systems. Results from the tests have been presented in this paper and shows accurate detection result of the car park occupancy. Feature detection stream is able to compensate the presence of image noise while edge detection stream is able to compensate weakness caused by low surrounding illumination.

\subsection{Ivan Ganchev and Mairtin O' Droma has} proposed et.at [6] "A cloud based intelligent car parking services for smart cities"------ In this the IOT sub system includes sensor layer, communication layer and application layer. The primary goal of the intelligent car parking system is to find, allocate and reserve the best available car parking lot for a user who is driving a car in a particular area and to provide instructions for reaching this lot. Sensor layer detecting the car lot occupancy. A car parking lot detection method is proposed based on carmatic threshold algorithm. An infostation based multi-agent system facilitating a car parking locator service is proposed. An access control system for reducing the waiting time proposed. At the application layer, an information centre provides cloud based service. An IOT management centre administrates the smart city via an IOT integrated service portal. A number of business services explore interfaces to the sensor layer. These includes a car parking locator service, car parking supervision service, car parking information service, GIS and GPS services, vehicle license patrolling, vehicle tracking service. At the communication layer various wireless technologies provide connection between the application and the sensor layer based on the ABC\&S(Always Best Connected and Best Served Communication paradigm. A 3-tier infostation based network architecture could be integrated in this layer to enable" anytime-anywhere-anyhow communication" among smart cities. Different sensing technologies could be utilized at the sensor layer such as Radio Frequency Identification (RFID), laser, infrared, radar, ultrasonic, CCTV, acoustic. RFID used for embedded parking solutions. CCTV with video image processing for detecting the status of parking lots. Communication layer includes $3 \mathrm{G}, 4 \mathrm{G}$, ZigBee, Wifi, wiMax, V2X,WSN, VANET. 3G/4G communication module used for car's tracking and tracing. Parking meter is an optimal element needed only for paid car parks. The architecture consists of a cloud tier, mobile apps tier, OSGI web servers tier. Cloud tier provides data storage and computing resources for the car parking service. It stores available car parking lots, car's location, user's location, profiles etc. The recent data is usually stored in Hadoop's Hbase it support real time queries. The OSGI web server, it acts as bridge between mobile application tier and cloud tier. Considering the great number of web applications running in this tier, it should support the deployment of new or updated application without stopping or restarting the web server. The OSGi provides an environment to modularize web applications into bundles. The bundles are registering itself in its environment. A distributed collecting system collects web server's log data and sends them to the cloud. In the mobile application tier, the first version of the car parking mobile application is developed for android mobile phones. When a user enters into a city an carmatic request is sent by the application to a OSGi car parking web server asking for available car parking lots. The server finds the best available car parking lots for this user based on their preferences specified in the user profile. Driving directions are returned to the user along with a detailed map.

\subsection{A Cloud-Based Smart-Parking System Based on Internet-of-Things Technologies---}

This paper provides a unique algorithm which increases the capability of the current cloud based smart parking system [5] and it also develops a network architecture based on the Internet of Things technology. This system helps the users to find a free parking space with minimal cost based on new performance metrics which is carmatic. This metrics will calculate the user spaces in each car park. To enhance the parking management, an intelligent parking system was developed which reduced the purpose of hiring people to maintain the parking system [1]. In this paper it proposes an effective cloud-based Smart parking system based on the Internet of Things. The data that includes the vehicle GPS location and distance between car parks and number of free parking space in car parks will be sent to the data center. Here the data center is presented as a cloud server which calculates the costs of a user parking request and this information is regularly updated and is made available to the vehicles in the network at any time. In this proposed system, each car park is an IoT network and it operates independently as a regular car park. This paper implements a system model with wireless access in an open-source physical computing platform based on Arduino with RFID technology. It uses smartphone that acts as a user interface between the cloud and the vehicles to check the feasibility of the proposed system [4].

2.10 A New "Smart Parking" System Based on Optimal Resource Allocation and Reservations----In this system a new smart parking system is implemented for cities. This system assigns and reserves a parking space for a user (driver) based on the users distance from the parking area and parking cost and also ensures that the overall parking capacity is effectively utilized. Their approach solves a Mixed Integer Linear Program (MILP) problem at each decision point in a time-driven sequence [3]. For each MILP there is a solution which gives an optimal allocation based on user's current state information and also supports random events such as new user requests and parking space availability. The allocation is updated at the next decision point which ensures that there is no parking slot reservation conflict and that no user is ever assigned a parking slot with higher than the current cost function value. This mechanism ensures a better response from the system along with assured reservations.

2.11 Faiz Shaikh1, Nikhilkumar B.S.2, Omkar Kulkarni3, Pratik Jadhav4, Saideep Bandarkar5-2015 has proposed A Survey on "Smart Parking" System-----People owning vehicles face parking problems in most metropolitan area, especially during peak hours. The difficulty roots from not knowing where the parking spaces are available at the given time, even if this is known; many vehicles may pursue a small number of parking spaces which in turn leads to serious traffic congestion. This paper focuses on different smart parking techniques developed to overcome said problem using various wireless sensor network and providing real-time data analysis 
from the sensors, some papers include system based on resource allocation and reservation of parking lot which have various problems in efficiently achieving the goals. The given paper would be useful for new researchers for study of various guided parking and information techniques and algorithms which are covered in this paper.

2.12 Prof. Yashomati R. Dhumal1, Harshala A. Waghmare2, Aishwarya S. Tole2, Swati R. Shilimkar2-2016 has proposed Android Based Smart Car Parking System-----The purpose of this system is to computerize the parking space reservation. Its talk about undertaking which introduces a miniature model of car parking that can direct and manage the number of cars that can be parked in given space at any given time based on availability of parking space after doing the registration by user using android application on his smart phone. Carmated parking is a strategy for parking and leaving cars utilizing detecting device i.e., sensors. The entering to or leaving from parking lot is also commanded by an android based application. This provide users to book parking spaces online in advance for given location and then park the vehicle with minimal fees. We have concentrated on some current systems and it shows that the current systems are not totally carmated and require a certain level of human interference and communication with the system. The difference between our system and existing systems is that we intend to make our system as less human independent by carmating whole parking area

\subsection{Vishwanath Y1, Aishwarya D Kuchalli2,} Debarupa Rakshit-2016 has proposed A survey paper on smart parking system based on internet of things a Smart Parking system---- It provides an optimal solution for parking problem in metropolitan cities. Due to rapid increase in vehicle density especially during the peak hours of the day, it is a difficult task for the drivers to find a parking space to park their vehicles. The aim of the paper is to resolve the above mentioned issue which provides the Smart Parking system. This system uses cloud computing and Internet of Things (IOT) technology. A suitable shortest path algorithm is used to find the minimum distance between the user and each car park in the system. Thus, the waiting time of the user is minimized.The paper also introduces the usage of android application using smart phone for the interaction between the Smart Parking system and the user. RFID technology is used in this system to avoid the human intervention which minimizes the cost.

\section{PROPOSED METHODOLOGY}

Shrewd Car Parking System Using Arduino UNO Designing, creating and delivering a main edge stopping innovation is called as Smart stopping. It is a vehicle stopping framework that helps drivers locate an empty spot. Utilizing the Ultrasonic sensors in each stopping space, it identifies the nearness or nonattendance of a vehicle. Brilliant Parking framework is demonstrated as a correct, strong and cost effective approach to guarantee that street clients know precisely where empty car parking spots are.

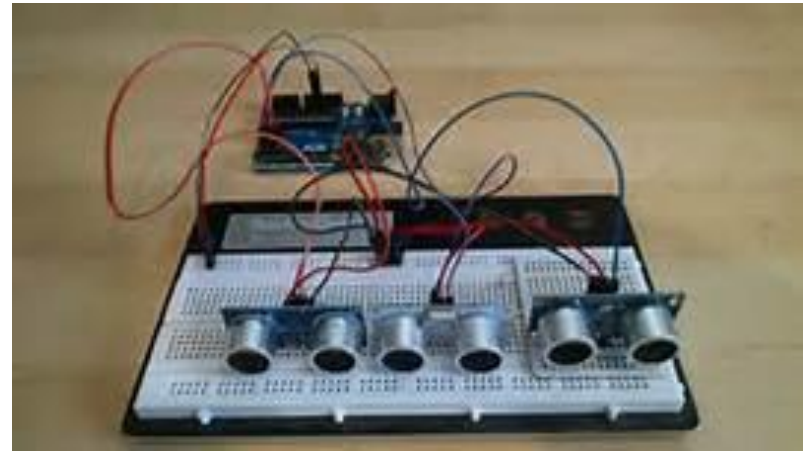

Figure 1: Proposed Architecture

As the populace expanded in the metropolitan urban areas, the utilization of the vehicles has also expanded. It causes issue for stopping which prompts movement clog, driver disappointment, and air contamination. When we visit the different open spots like shopping centres, multiplex film lobby and lodgings amid the celebration time or ends of the week it makes all the more stopping issue. In the current research found that a driver takes almost 8 minutes to stop his vehicle since he invest more energy in looking the parking area. This seeking prompts 30 to $40 \%$ of activity blockage. This project perceives how to diminish the stopping issue and to do secured stopping utilizing the shrewd stopping under Slot Allocation strategy with the assistance of Arduino UNO. The primary commitment of our proposed frameworks is to discover status of the stopping territory and give secured stopping. In the course of recent years, movement experts in numerous urban areas have built up a model called Parking Guidance and Information (PGI) framework for good stopping administration. PGI frameworks, tells about the dynamic data of stopping in the controlled region and aides the clients to the empty stopping spaces.

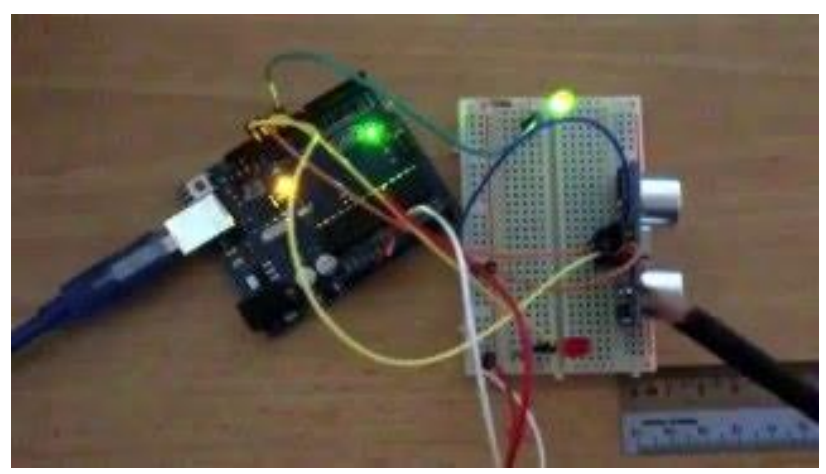

Figure 2: Demonstration of Parking Slot

\subsection{The elements of the proposed framework are:}

- Drivers find accessible parking spots close to them.

- Less number of drivers seeking to stop, in this way decreases the movement clog.

- Avoids air contamination and a dangerous atmospheric deviation.

- Scalable, hearty and solid.

- Reduces the driver stretch and enhances the urban range.

- Provides devices to streamline the parking spot administration accurately discover the vehicle inhabitancies progressively. 


\subsection{Disgraceful Parking :}

In the event that an car is parked such that it involves two parking slots instead of one, this is called disgraceful parking. Parking can happen when a driver is not cautious about another driver's rights. This is handled by the advancement of robotized keen car parking framework.

\section{RESULTS AND DISCUSSION}

1. It guarantees snappy and computerized parking and simple recovery of vehicles.

2. Up to 3 cars can be effectively and securely parked in the outlined model.

3. The surface space required is identical to the parking spot of two cars as it were.

4. Most reasonable for parking in workplaces, shopping centers and comparable spots.

5. Low support levels are required by the framework.

6. Sensors utilized have high affectability and are anything but difficult to deal with.

7. Minimal effort framework, giving most extreme computerization.

8. It doesn't require observable pathway operation.

9. Cordial reorientation of cars for driving in and out.

10. Security of vehicle.

\subsection{Hardware Connections:}

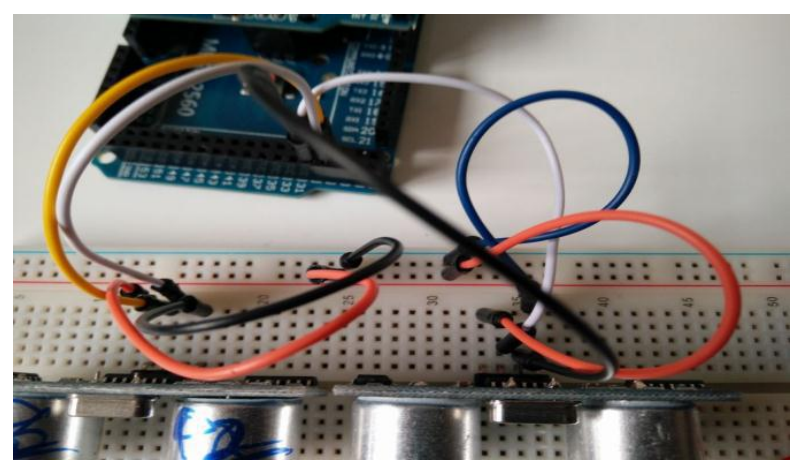

Figure 3: Working model of Smart Car Parking System

- Connect the Vcc stick to the positive rail on your breadboard.

- Connect the Gnd stick to the negative rail on your breadboard.

- Connect the Trig stick to any advanced stick on the arduino.

- Connect the Echo stick to any computerized stick on the arduino.

- Finally, interface the positive rail of the breadboard to $5 \mathrm{~V}$ stick on the arduino and the negative rail of the breadboard to the Gnd stick on the arduino.

\section{CONCLUSIONS}

Our project detects the empty slots and helps the drivers to find parking space in unfamiliar city. The average waiting time of users for parking their vehicles is effectively reduced in this system. The optimal solution is provided by the proposed system, where most of the vehicles find a free parking space successfully. Our preliminary test results show that the performance of the Arduino UNO based system can effectively satisfy the needs and requirements of existing car parking hassles thereby minimizing the time consumed to find vacant parking lot and real time information rendering. This smart parking system provides better performance, low cost and efficient large scale parking system. When car enters the parking area, the driver will park the car in the nearest empty slot when slot is occupied the LED light glows and when slot is empty LED lights are turned off carmatically indicating that the parking slot is empty to be occupied. It also eliminates unnecessary travelling of vehicles across the filled parking slots in a city.

\section{FUTURE ENHANCEMENT}

In future works, this framework can be enhanced by including different applications, For Example, internet booking by utilizing GSM. The driver or client can book their parking area at home or while in transit to the shopping center. This can diminish the season of the client to seeking the empty parking area. As a further review, distinctive sensor frameworks can be added to enhance this framework to distinguish the question and guide the driver or clients speediest. We will attempt to decrease the mechanical structure and attempt to make it ecofriendly.

\section{ACKNOWLEDGEMENT}

We thank our HOD mam and all other staff member for their support.

\section{REFERENCES}

[1] Thanh Nam Pham1, Ming-Fong Tsai1, Duc Bing Nguyen1, Chyi-Ren Dow1 and Der-Jiunn Deng2. "A Cloud- Based Smart-Parking System Based on Internetof-Things Technologies". IEEE Access, volume 3, pp. 1581 - 1591, september 2015

[2] M. Fengsheng Yang, Android Application Development Revelation, China Machine Press, 2010.

[3] Yanfeng Geng and Christos G. Cassandras. "A New Smart Parking System Based on Optimal Resource Allocation and Reservations". IEEE Transaction on Intelligent Transportation Systems, volume 14, pp. 1129 -1139, April 2013

[4] Cui Shiyao, Wu Ming, Liu Chen, Rong $\mathrm{Na}$. "The Research and Implement of the Intelligent Parking Reservation Management System Based on ZigBee Technology". Measuring Technology and Mechatronics Automation (ICMTMA) , pp. 741-744, January 2014.

[5] K.Ashokkumar a, Baron Sam , R.Arshadprabhu , Britto. "Cloud Based Intelligent Transport System". Procedia Computer Science, volume 50, pp. 58-63, 2015.

[6] Z. Ji, I. Ganchev, M. O'Droma, and X. Zhang, “A cloud-based intelligent car parking services for smart cities," in Proc. 31st URSI General Assembly Sci. Symp. (URSI GASS), Aug. 2014.

[7] Hamada R.H.AI-Absi,Patrick Sebastian ,"Vision-Based Automated Parking System "in 10th International Conference on Information science, 2010

[8] Sarfraz nawaz, Christos Efstratiou, Celia Mascolo,"Parksense: A smartphone based sensing system foron street parking" in Cambridge university 
[9] B. K. Konstantinos Domdouzis and C. Anuba., "An experimental study of the effects of different medium on the performance of rfid system," vol. 21. Advanced Engineering Informatics, 2011.

[10] K. Finkenzeller, Fundamentals and Applications in Contactless Smart Cards and Identification. John Wiley and Sons Ltd, 2003.

[11] K. M. R. Sudeep Dogra, "Radio frequency identification(RFID) applications: A brief Introduction, advanced engineering informatics." The IUP journal of Electrical and Electronics Engineering, 2011, p. 3.

[12] J. Dongjiu Geng, Yue Suo, Yu Chen, Jun Wen, Yongqing Lu, Remote Access and Control System Based on Android Mobil Phone, vol.2. Journal of Computer Applications, 2011, pp. 560-562
[13] M.A.R. Sarkar, A.A. Rokoni, M.O. Reza, M.F. Ismail, "Smart Parking system with image processing facility", I.J. Intelligent Systems and Applications, 2012, vol. 3, pp. 41-47.

[14] Z. L. Wang, C. H. Yang, and T. Y. Guo, "The design of an autonomous parallel parking neuro-fuzzy controller for a car-like mobile robot," in Proceedings of the SICE Annual Conference, Taipei, 2010, pp. 2593-2599.

[15] J. Dongjiu Geng, Yue Suo, Yu Chen, Jun Wen, Yongqing Lu, Remote Access and Control System Based on Android Mobil Phone, vol.2. Journal of Computer Applications, 2011, pp. 560-562 